\title{
The location of the last stable orbit in Kerr spacetime
}

\author{
Leo C. Stein $\left(\mathbb{D}^{1, *}\right.$ and Niels Warburton (D) $^{2, \dagger}$ \\ ${ }^{1}$ Department of Physics and Astronomy, The University of Mississippi, University, MS 38677, USA \\ ${ }^{2}$ School of Mathematics and Statistics, University College Dublin, Belfield, Dublin 4, Ireland.
}

\begin{abstract}
Black hole spacetimes, like the Kerr spacetime, admit both stable and plunging orbits, separated in parameter space by the separatrix. Determining the location of the separatrix is of fundamental interest in understanding black holes, and is of crucial importance for modeling extreme mass-ratio inspirals. Previous numerical approaches to locating the Kerr separatrix were not always efficient or stable across all of parameter space. In this paper we show that the Kerr separatrix is the zero set of a single polynomial in parameter space. This gives two main results. First, we thoroughly analyze special cases (extreme Kerr, polar orbits, etc.), finding strict bounds on the limits of roots, and unifying a number of results in the literature. Second, we pose a stable numerical method which is guaranteed to quickly and robustly converge to the separatrix. This new approach is implemented in the Black Hole Perturbation Toolkit, and results in a $\sim 45 \times$ speedup over the prior robust approach.
\end{abstract}

\section{INTRODUCTION}

The existence of unstable and plunging orbits for test body motion in general relativity is one of the key differences in celestial mechanics between Newtonian and Einstein gravity. In the strong gravitational field around black holes, a region of the parameter space appears where stable bound orbits are no longer possible. In this region test bodies either plunge directly into the black hole or are on unstable orbits to which any slight perturbation will trigger the body to plunge. This has important consequences in astrophysics. For example, the inner edge of a black hole accretion disk is set by the location of the innermost stable circular orbit (ISCO). The relation between the ISCO radius and the black hole's spin is exploited to make measurements of the rotation rate of astrophysical black holes [1].

The ISCO delineates one edge of a more general structure called the 'separatrix' that divides the stable region of the parameter space from the unstable/plunging region. This separatrix is particularly important for the physics of extreme mass-ratio inspirals (EMRIs) [2, 3], key sources for the future space-based gravitational wave detector LISA. The event rate of these binaries is strongly influenced by the location of the separatrix, with highly spinning massive black holes more likely to capture stellar mass compact objects on prograde orbits [4]. Once the secondary is captured its orbit will decay through gravitational wave emission until it reaches the separatrix and plunges into the massive black hole. Consequently, knowledge of the location of the separatrix is a key ingredient in models of these binaries [5-12]. The region of parameter space near the separatrix is also interesting as it is here that the well known relativistic orbital precession is taken to the extreme, with arbitrary large precession possible when approaching the separatrix [13].

\footnotetext{
*1cstein@olemiss.edu

$\dagger$ niels.warburton@ucd.ie
}

Calculating the location of the separatrix for generic orbits that could be eccentric or inclined is non-trivial. For certain limiting cases the location can be found analytically, but in general numerical solutions must be found. There are a variety of methods in the literature [4, 9] to find the separatrix for generic orbits but these are not always efficient or stable across the entire parameter space. In this work we show that the separatrix is an algebraic variety, and derive a single polynomial, of degrees $(12,12,12,4)$ in the indeterminates $\left(a, p, e, x^{2}\right)$, which are the orbital parameters detailed below. The roots of this polynomial give the location of the separatrix. This has two benefits: (i) it is easy to analyze the limiting cases (equatorial motion, extreme Kerr, etc.), and (ii) we can apply rapidly convergent methods for finding the roots of polynomials. We analyze many limits and detail several numerical schemes, with a full implementation provided in the Black Hole Perturbation Toolkit [14].

The organization of this paper is as follows. Sec. II discusses time-like geodesic motion in Kerr spacetime focussing on bound orbits. Sec. III defines the separatrix and other special orbits and derives the separatrix polynomial. We look at solutions to the separatrix polynomial in interesting limiting cases in Sec. IV. Finally, in Sec. V we discuss numerical methods for solving the separatrix polynomial. In the appendices we give some additional details, including results for special orbits such as the innermost bound spherical orbit. We also present an alternative robust method for numerically locating the separatrix in Appendix D. Throughout this article we use geometrized units such that the speed of light and the gravitational constant are equal to unity. We also use standard Boyer-Lindquist coordinates $(t, r, \theta, \varphi)$ and use the metric signature $(-+++)$.

\section{TIME-LIKE GEODESICS IN KERR SPACETIME}

Given any spacetime, let us denote the trajectory of a timelike (non-spinning) test body of mass $\mu$ by a curve $x^{\alpha}(\tau)$ where $\tau$ is the proper time as measured along the 
world line. The four-velocity of the body is given by $u^{\alpha}=d x^{\alpha} / d \tau$ where for timelike motion we have (with our choice of metric signature) $u^{\alpha} u_{\alpha}=-1$. The test body's trajectory is governed by the second-order differential equation $u^{\beta} \nabla_{\beta} u^{\alpha}=0$ where $\nabla_{\beta}$ is the covariant derivative with respect to the background geometry.

Hereafter we focus on motion about a Kerr black hole. The Kerr spacetime is parameterized by the black hole mass, $M$, and its spin $a$, where $a=J / M$ with $J$ the angular momentum of the black hole. We choose $J \geq 0$ so that $0 \leq a \leq M$. For motion about a Kerr black hole the Killing symmetries of the spacetime give rise to conserved quantities. Two of these, the orbital energy and (azimuthal) angular momentum, are associated with isometries of the metric, with associated Killing vector fields $\left(\partial_{t}\right)^{\alpha}$ and $\left(\partial_{\varphi}\right)^{\alpha}$. The third, the Carter constant, is related to a hidden symmetry associated with a Killing tensor $\mathcal{Q}^{\alpha \beta}$ of the spacetime, satisfying $\nabla_{(\alpha} \mathcal{Q}_{\beta \gamma)}=0$. With these constants of motion, and the conserved mass of the test body, the geodesic equations in Boyer-Lindquist coordinates can be written in first-order form:

$$
\begin{aligned}
\Sigma^{2}\left(\frac{d r}{d \tau}\right)^{2} & =R(r) \\
\Sigma^{2}\left(\frac{d \theta}{d \tau}\right)^{2} & =\Theta(\theta) \\
\Sigma \frac{d \varphi}{d \tau} & =\frac{a}{\Delta}\left(2 r \mathcal{E}-a \mathcal{L}_{z}\right)+\frac{\mathcal{L}_{z}}{\sin ^{2} \theta} \\
\Sigma \frac{d t}{d \tau} & =\frac{\left(r^{2}+a^{2}\right)^{2} \mathcal{E}-2 a r \mathcal{L}_{z}}{\Delta}-a^{2} \mathcal{E} \sin ^{2} \theta,
\end{aligned}
$$

where $\Sigma \equiv r^{2}+a^{2} \cos ^{2} \theta, \Delta \equiv r^{2}-2 M r+a^{2}$ and

$$
\begin{aligned}
R(r)= & -\beta r^{4}+2 r^{3}-\left(a^{2} \beta+\mathcal{L}_{z}^{2}\right) r^{2} \\
& +2\left(a \mathcal{E}-\mathcal{L}_{z}\right)^{2} r-\mathcal{Q} \Delta \\
\Theta(\theta)= & \mathcal{Q}-\cos ^{2} \theta\left\{a^{2} \beta+\frac{\mathcal{L}_{z}^{2}}{\sin ^{2} \theta}\right\}
\end{aligned}
$$

where $\beta=\left(1-\mathcal{E}^{2}\right)$. In the above equations and hereafter $\mathcal{E}, \mathcal{L}_{z}$, and $\mathcal{Q}$ denote the specific energy, angular momentum and Carter constant, respectively. These are related to the tangent $u_{\alpha}=\mu^{-1} p_{\alpha}$ and the Killing vectors and tensor via

$$
\mathcal{E} \equiv-\left(\partial_{t}\right)^{\alpha} u_{\alpha}, \quad \mathcal{L}_{z} \equiv\left(\partial_{\varphi}\right)^{\alpha} u_{\alpha}, \quad \mathcal{Q} \equiv \mathcal{Q}^{\alpha \beta} u_{\alpha} u_{\beta},
$$

where we follow the convention for the Carter tensor in Boyer-Lindquist coordinates ordered $(t, r, \theta, \varphi)$,

$$
\begin{aligned}
\mathcal{Q}^{\alpha \beta}= & \operatorname{diag}\left(-a^{2} \cos ^{2} \theta, 0,1, \cot ^{2} \theta\right)^{\alpha \beta} \\
& -\left(a^{2} \cos ^{2} \theta\right) g^{\alpha \beta} .
\end{aligned}
$$

Introducing the Mino time parameter $\lambda$ defined by [15],

$$
\frac{d \tau}{d \lambda}=\Sigma
$$

the system of ordinary differential equations (ODEs) can be decoupled, so one would instead integrate the system

$$
\begin{aligned}
& \frac{d r}{d \lambda}= \pm_{r} \sqrt{R(r)}, \\
& \frac{d \theta}{d \lambda}= \pm_{\theta} \sqrt{\Theta(\theta)} .
\end{aligned}
$$

Then with solutions for $r(\lambda)$ and $\theta(\lambda)$ in hand, one can integrate for $\varphi(\lambda), t(\lambda)$, and the one-to-one function $\tau(\lambda)$ (and thus recover $x^{\alpha}(\tau)$ if so desired).

The upper/lower signs in Eqs. (10) and (11) are to be chosen when the particle is outgoing/ingoing in the radial equation, or downgoing/upgoing in the polar equation. A sign flip occurs in an equation when the particle passes a turning point of the radial or polar motion. Numerically integrating this type of equation is inconvenient, as it requires accurate numerical identification of turning points; and moreover, when passing through such a turning point, the source in the differential equation fails to be Lipschitz continuous, becoming infinitely steep as one approaches the turning point. Failing the Lipschitz condition, the Picard-Lindelöf theorem says one can no longer prove existence and uniqueness of solutions to the ODEs (this is not a problem for the second order geodesic equations). Therefore a reparameterization is necessary.

\section{A. Parameterization for bound orbits}

Hereafter we shall be concerned with bound orbits about a Kerr black hole. For such orbits the radial motion is confined within the region $r_{p} \leq r \leq r_{a}$, where $r_{p}$ and $r_{a}$ are the minimum (pericenter) and maximum (apocenter) radii obtained during the orbital motion, respectively. Similarly, the polar motion is confined within the region $\theta_{\min } \leq \theta \leq \theta_{\max }=\pi-\theta_{\min }$. An orbit in the equatorial plane has $\theta=\theta_{\min }=\pi / 2$.

There are infinitely many ways to parametrize geodesic motion in Kerr spacetime. For bound orbits it is convenient to change from the set $\left(\mathcal{E}, \mathcal{L}_{z}, \mathcal{Q}\right)$ to a Keplerianinspired choice. One such choice for the radial motion is the quasi-Keplerian parameterization,

$$
r=\frac{p M}{1+e \cos \psi},
$$

where $p$ is the dimensionless semi-latus rectum, $0 \leq e<1$ is the orbital eccentricity, and $\psi$ is a monotonically increasing radial phase parameter. The minimum (pericenter) and maximum (apocenter) radii occur at

$$
r_{p}=\frac{p M}{1+e}, \quad r_{a}=\frac{p M}{1-e},
$$

which can be inverted to give

$$
p=\frac{2 r_{a} r_{p}}{M\left(r_{a}+r_{p}\right)}, \quad e=\frac{r_{a}-r_{p}}{r_{a}+r_{p}} .
$$




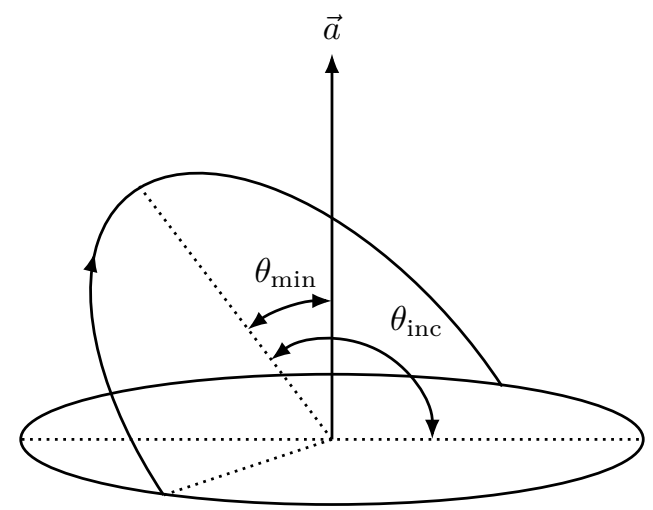

FIG. 1. We parameterize orbital inclination by $x=\cos \theta_{\text {inc }}$, see Eq. (16). For a prograde orbit, $\theta_{\text {inc }}+\theta_{\min }=\pi / 2$, whereas for a retrograde orbit (shown here), $\theta_{\text {inc }}-\theta_{\text {min }}=\pi / 2$. Using $x$ lifts the degeneracy that a single value of $\theta_{\min }$ maps to both prograde and retrograde orbits.

Using the parameterization of Eq. (12) will avoid the issue of sign flipping at turning points, since the radial phase $\psi$ is monotonically increasing. Further, one can show that this parameterization analytically cancels the zeroes in $R(r)$ at $r_{p}$ and $r_{a}$, thus making the ODE satisfy the Lipschitz condition.

A similar approach works for the polar angle. Defining $z=\cos \theta$, we can write

$$
z=z_{m} \cos \chi
$$

where $z_{m}=\cos \theta_{\min }$, so $\pm z_{m}$ are the maximum/minimum values achieved by $\cos \theta$, and $\chi$ is a monotonically increasing phase angle. This parameterization similarly solves the sign choice and Lipschitz continuity issues.

One drawback of using $z_{m}$ as an "inclination" parameter is that it does not distinguish between prograde and retrograde orbits. This distinction must be implemented by making $a<0$ for retrograde orbits. Besides $z_{m}$, there are many common parameterizations for the inclination angle in the literature. Because of the plethora of inclination parameterizations in the literature care must be taken when comparing results between different works. In this work we primarily use

$$
x=\sin \left[\operatorname{sign}\left(\mathcal{L}_{z}\right) \theta_{\mathrm{min}}\right]=\cos \theta_{\mathrm{inc}} .
$$

This has the nice property that the orbital parameters smoothly vary from prograde equatorial motion $(x=1)$ to retrograde equatorial motion $(x=-1)$, without having to flip the sign of $a$. The relationship between $\theta_{\min }$ and $\theta_{\text {inc }}$ is diagrammed in Fig. 1 . The parameters $x$ and $z_{m}$ satisfy the polynomial relationship $x^{2}+z_{m}^{2}=1$, which is significant in that any polynomial results developed with $x^{2}$ will also be polynomial in $z_{m}^{2}$. Another commonly used inclination angle is $\cos \iota=\mathcal{L}_{z} / \sqrt{\mathcal{L}_{z}^{2}+\mathcal{Q}}$. Using $\cos \iota$ or $\sin \iota$ also turns out to yield polynomial relations below.
When parameterizing an orbit by $(p, e, x)$, it is crucial to know how to convert back and forth between these parameters and the constants $\left(\mathcal{E}, \mathcal{L}_{z}, \mathcal{Q}\right)$. The bijective relationship [16] between $(p, e, x) \leftrightarrow\left(\mathcal{E}, \mathcal{L}_{z}, \mathcal{Q}\right)$ is well known for bound orbits in Schwarzschild spacetime [17] as well as equatorial orbits [13], spherical orbits [18], and generic orbits in Kerr spacetime [19]. Unfortunately, not all of $(p, e, x)$ space maps to stable bound orbits, or even to physically realizable motion. Finding the separatrix between the stable and plunging orbits is the subject of the remainder of this paper.

\section{B. Orbit naming conventions}

Certain classes of orbital configurations are simpler to analyze than others. These special classes of orbits are as follows:

1. Equatorial orbits. These lie in the equatorial plane $(\theta=\pi / 2)$ and have $|x|=1$.

2. Polar orbits. These orbits have $x=0$ which corresponds to $\mathcal{L}_{z}=0$. They intersect the axis of symmetry of the black hole.

3. Spherical orbits. These orbits have $e=0$ and $|x| \neq 1$. These orbits have fixed Boyer-Lindquist radius and librate in the polar direction.

4. Circular orbits. These orbits have $e=0$ and $|x|=1$. These orbits lie in the equatorial plane and have a fixed Boyer-Lindquist radius.

5. Parabolic trajectories. These have $e=1$ and $\mathcal{E}=1$, sending apocenter to infinity, so are marginally bound and technically not orbits.

If an orbital configuration does not fall into any of the above categories we refer to it as a 'generic' orbit.

\section{THE SEPARATRIX AND OTHER SPECIAL ORBITS}

The separatrix is the locus of points in the $(p, e, x)$ parameter space which separates bound orbital motion from trajectories that plunge into the black hole. At fixed $a$, the separatrix forms a two dimensional surface bounded within $0 \leq e \leq 1$ and $-1 \leq x \leq 1$ in $(p, e, x)$ space. For parameters in this range we define the location of the separatrix as $p_{\text {sep }}(a, e, x)$.

In the literature, orbits with parameters along the separatrix are referred to as last stable orbits (LSOs) or marginally stable orbits [20]. Orbits along the separatrix with $e=0$ are referred to as the innermost stable spherical orbit (ISSO). If $|x|=1$ this orbit is usually called the innermost stable circular orbit (ISCO) instead. At the other extreme there are parabolic orbits with $e=1$. These orbits have $\mathcal{E}=1$ and are marginally bound. 
As the orbital parameters approach the separatrix the amount of azimuthal precession diverges [13, 17]. This gives rise to the 'zoom-whirl' behavior of orbits near the separatrix [13]. In the limit the whirl phase becomes infinitely long and there is a mapping between a spherical orbit at the whirl radius and the separatrix parameters see Ref. [21] for the equatorial case and Appendix D for the extension to generic orbits. Through this relation the marginally bound orbits are related to spherical orbits with $\mathcal{E}=1$. These orbits are called the innermost bound spherical orbit (IBSO), or if in the equatorial plane, the innermost bound circular orbit (IBCO). The majority of this work is about the separatrix in general but we give additional results for the IBSO in Appendix C.

For the remainder of this work we set $M=1$ for the sake of brevity.

\section{A. The separatrix polynomial}

Bound radial motion occurs between two roots of the radial polynomial $R(r)$. The four roots are traditionally labeled as $r_{1} \geq r_{2} \geq r_{3} \geq r_{4}$ (when they are all real), in the factorization

$$
R(r)=\left(1-\mathcal{E}^{2}\right)\left(r_{1}-r\right)\left(r-r_{2}\right)\left(r-r_{3}\right)\left(r-r_{4}\right) .
$$

The signs above are chosen since bound motion happens in the range $r_{p}=r_{2} \leq r \leq r_{1}=r_{a}$, and $\mathcal{E}^{2}<1$ for bound motion.

When $r_{2}>r_{3}$, there is a simple root, $R\left(r_{2}\right)=0$ and $R^{\prime}\left(r_{2}\right) \neq 0$, and thus a 'restoring force' to keep the particle from plunging. By contrast, if we have a root with higher multiplicity, $r_{2}=r_{3}$ (or $r_{1}=r_{2}=r_{3}$ for circular orbits), then the derivative of the radial polynomial vanishes, $R^{\prime}\left(r_{2}\right)=0$. This means there is no 'restoring force' at pericenter, so an infinitesimal perturbation can make the orbit plunge.

This gives the condition for the separatrix in parameter space: the set of parameters where these roots degenerate, solving the equation $r_{2}(p, e, x)=r_{3}(p, e, x)$.

The root $r_{2}=p M /(1+e)$ is a simple function of $p$ and $e$. The root $r_{3}$ is much more complicated, though it is possible to express it in terms of nested radicals (this earlier method is described in Sec. V A). We however pursue an approach which yields the separatrix polynomial $S(a, p, e, x)$, where the separatrix lies along roots of the polynomial equation $0=S(a, p, e, x)$.

To find the separatrix polynomial, we start by posing the location of the separatrix as the simultaneous solutions of the following system of equations:

$$
\left\{\begin{array}{l}
0=\Theta\left(z_{m}\right) \\
0=R\left(\frac{p}{1-e}\right) \\
0=R\left(\frac{p}{1+e}\right) \\
0=R^{\prime}\left(\frac{p}{1+e}\right) .
\end{array}\right.
$$

It is important to note here that every equation in system (18) is a rational polynomial in all the following indeterminates: $\left(a, p, e, z_{m}, \mathcal{E}, \mathcal{L}_{z}, \mathcal{Q}\right)$. Since $\Theta$ is a function only of the square $z_{m}^{2}=1-x^{2}$, this system is still a system of rational polynomials in $x$ instead of $z_{m}$. The same property is true if we use $\sin \iota$ or $\cos \iota$ instead of $z_{m}$. We overload the symbol $\Theta$ to mean the appropriate function of each variable, so we write $\Theta(x)$.

Now if we avoid vanishing denominators (the point is said to be in general position), we can clear denominators to form a system of polynomials in these indeterminates. This system is

$$
\left\{\begin{array}{l}
0=x^{2} \Theta(x) \\
0=(1-e)^{4} R\left(\frac{p}{1-e}\right) \\
0=(1+e)^{4} R\left(\frac{p}{1+e}\right) \\
0=(1+e)^{3} R^{\prime}\left(\frac{p}{1+e}\right) .
\end{array}\right.
$$

The simultaneous solution of a system of polynomial equations forms an algebraic variety, the fundamental object of algebraic geometry. Even before modern algebraic geometry, in classical elimination theory it was known that one could eliminate indeterminates from such a system at the expense of raising the polynomial degree of the remaining system. The classic method is based on generalizations and improvements to Dixon's resultant [22] (see e.g. [23]). The more modern algebraic geometry approach would construct a Gröbner basis for the ideal of the ring of polynomials vanishing on the variety defined by system (19).

The upshot is that with these four equations, we can eliminate the three indeterminates $\left(\mathcal{E}, \mathcal{L}_{z}, \mathcal{Q}\right)$, and be left with the single separatrix polynomial $S(a, p, e, x)$. As noted earlier, if one desires, the same approach works in terms of $z_{m}, \cos \iota$, or $\sin \iota$ instead of $x$. This can be accomplished with a computer algebra system such as MathematicA with a bit of guidance. The separatrix polynomial is $12^{\text {th }}$ degree in $p, a, e$ and $4^{\text {th }}$ degree in $x^{2}$, so is a bit cumbersome. After clearing some unwanted denominators introduced by elimination, we present $S$ as

$$
S=\sum_{n=0}^{12} S_{n} p^{n}
$$

where the $S_{n}$ are polynomials in $a, e$, and $x^{2}$ that we tabulate in App. A. We make the algebraic derivation and machine-readable expressions available in the companion MATHEMATICA notebook distributed with this article [24].

Figure 2 gives an overview of the set of points satisfying $S=0$. The polynomial $S$ is even in $x$, which is reflected in the reflection symmetry in the figure. Correspondingly, the polynomial is satisfied at both the prograde and retrograde values of $p$ associated to a particular $x^{2}$.

The separatrix itself is just one of the "leaves" of the solutions seen in Fig. 2, specifically the one for which $p$ decreases with increasing $x$, as prograde orbits exist closer than retrograde ones. Note also that there are additional 


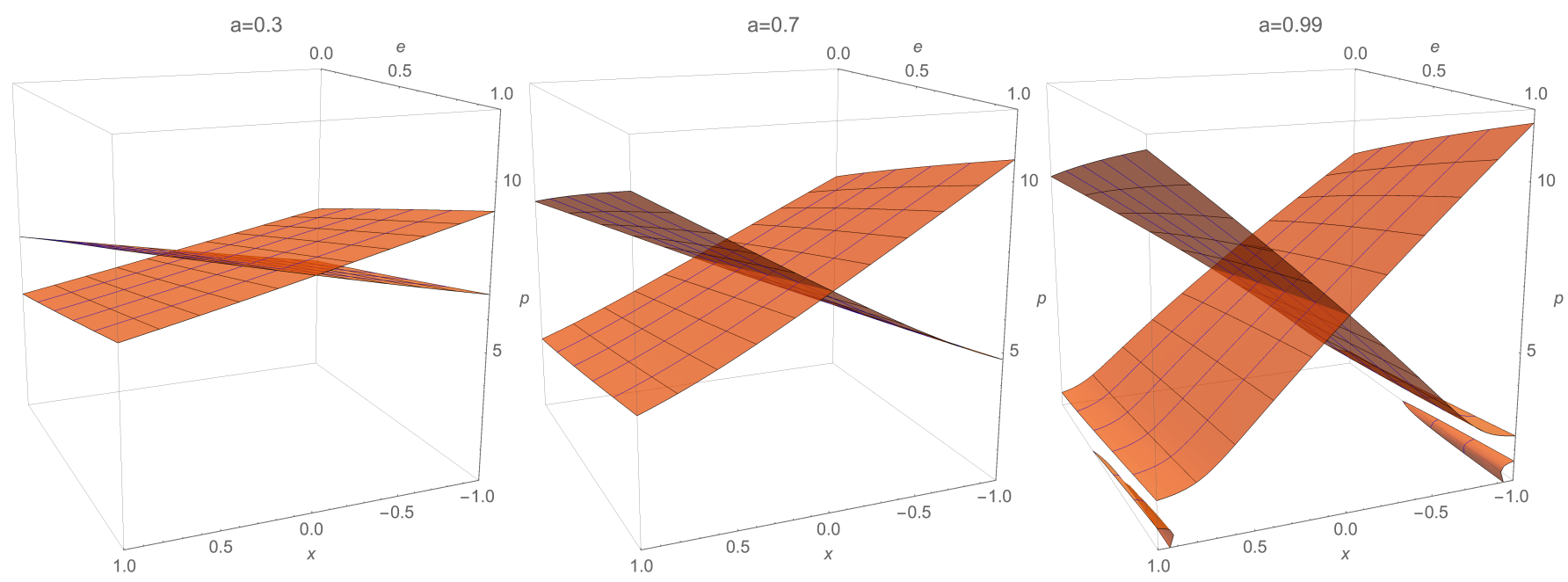

FIG. 2. Overview of solutions of the separatrix polynomial $S=0$ at selected values of spin. At each value of $(a, e, x)$, there are solutions for both the prograde and retrograde values of the separatrix $p_{\text {sep }}$ for the corresponding value of $x^{2}$. There may also be unphysical solutions, as seen in the rightmost panel, appearing at smaller values of $p$. The physical branch is the one sloping "downward" in $x$, i.e. $p_{\text {sep }}$ decreases as $x$ increases.

unphysical solutions which appear at smaller $p$ at high spin and eccentricity - more on this in Sec. IV B 1.

One might hope that the separatrix polynomial could be factorized into a lower-degree polynomial for each "leaf" of the solution set. Taking a more global view shows that this is impossible. In Fig. 3 we show a view of the affine variety (i.e. the set of solutions) that extends to unphysical values of $e<0$. There we see that at $e=-1$, the prograde and retrograde "leaves" are smoothly connected, so they are part of the same algebraic set.

The high degree of the separatrix polynomial makes finding analytic solutions for generic orbits unfeasible. Instead we now concentrate on analytic results for interesting limiting cases. We then present techniques for numerically computing the location of the separatrix for generic orbits.

\section{LIMITING CASES}

The complete information about the separatrix is contained in the single polynomial $S(a, p, e, x)$. This makes it very expedient to take various simplifying limits, wherein the degree of the polynomial reduces and thus simplifies.

\section{A. Schwarzschild}

As Schwarzschild spacetime is spherically symmetric, the separatrix must be independent of $x$. If we set $a=0$, we get the enormous simplification,

$$
S(a=0)=p^{10}(p-6-2 e)^{2} .
$$

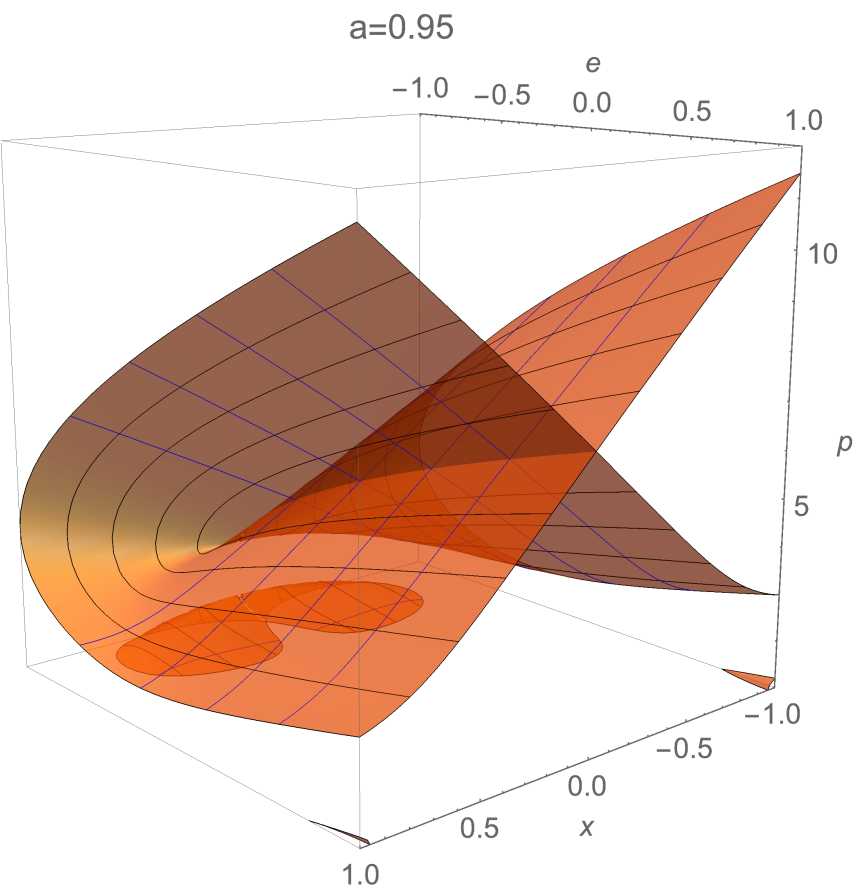

FIG. 3. Extended view, past the physical region, to show that the prograde/retrograde branches smoothly join at $e=-1$. Since they are part of the same surface, there is no possibility of a factorization for $S$ to give a lower-degree polynomial to describe only one of the two branches.

Thus we see that the Schwarzschild separatrix lies at $p_{\mathrm{sep}}^{\mathrm{Schw}}=6+2 e[17]$. 


\section{B. Equatorial}

Equatorial orbits have $x^{2}=1$, with the sign encoding prograde/retrograde motion. This corresponds to $\mathcal{Q}=0$ and $\sin \iota=0$. In this case we also get a significant factorization,

$$
S\left(x^{2}=1\right)=p^{8} S_{\text {equat }}(a, p, e),
$$

where the nontrivial quartic polynomial is

$$
\begin{aligned}
S_{\text {equat }}(a, p, e)= & a^{4}\left(-3-2 e+e^{2}\right)^{2} \\
& +p^{2}(-6-2 e+p)^{2} \\
& -2 a^{2}(1+e) p\left(14+2 e^{2}+3 p-e p\right) .
\end{aligned}
$$

This is the same as Eq. (B7) in [8] which is itself a simplified form of Eq. (23) in [13]. As Eq. (23) is quartic in $p$, there is an explicit solution by radicals for $p_{\text {sep }}^{ \pm \text {equat }}(a, e)$.

Because of monotonicity, the global extrema are at $a=1, x= \pm 1$. Along the extremal spin limit $a=1$, the quartic is

$$
\begin{aligned}
S_{\text {equat }}(1, p, e)= & (1+e-p)^{2} \\
& \times\left((-3+e)^{2}-2(5+e) p+p^{2}\right) .
\end{aligned}
$$

The global minimum is at $a=1, e=0, x=+1$, with value $p_{\mathrm{sep}}^{\min }=1$. The global maximum is at $a=1, e=1, x=-1$, with value $p_{\text {sep }}^{\max }=6+4 \sqrt{2} \approx 11.66$. These two extremes bracket all values for physical solutions of the separatrix,

$$
1 \leq p_{\mathrm{sep}}(a, e, x) \leq 6+4 \sqrt{2} .
$$

In the equatorial plane the separatrix is a one dimensional curve in the $(p, e)$ parameter space. A parameterization for this curve can be found by noting the connection between the separatrix and the unstable 'whirl' radius, $r_{u}=p /(1+e)$ of a homoclinic orbit [21]. This radius varies in the range $r_{\text {ibco }} \leq r_{u} \leq r_{\text {isco. The second inequal- }}$ ity implies that $r_{u}\left(r_{u}-2\right) a+a^{3} \geq 0$. Simultaneously solving the set of equations $\left\{S_{\text {equat }}=0, r_{u}=p /(1+e)\right\}$ with the above constraint gives

$$
\begin{aligned}
& e_{\text {sep }}=\frac{-r_{u}^{2}+6 r_{u}-8 a r_{u}^{1 / 2}+3 a^{2}}{r_{u}^{2}-2 r_{u}+a^{2}}, \\
& p_{\text {sep }}=\frac{4 r_{u}\left(r_{u}^{1 / 2}-a\right)^{2}}{r_{u}^{2}-2 r_{u}+a^{2}} .
\end{aligned}
$$

These equations agree with the results in Ref. [21].

\section{Number of real equatorial roots and brackets}

The quartic $S_{\text {equat }}(p)$ has four roots, but they are not all real throughout the unit square in $(a, e)$ space. We can find the positions of degenerate roots in parameter space by examining the discriminant of $S_{\text {equat }}$ when treated as a polynomial in $p$. This discriminant is

$$
\begin{aligned}
& \Delta_{\text {equat }}(a, e)=2^{20} a^{6}\left(a^{2}-1\right)(1+e)^{4} \\
& \quad \times\left(a^{2}(-3+e)^{3}(1+e)-(-1+e)(3+e)^{3}\right) .
\end{aligned}
$$

Degeneracies occur when the discriminant vanishes. At $a=0$, the prograde and retrograde separatrices coalesce and are thus degenerate. The degeneracy at $e=-1$ is unphysical but can be seen in Fig. 3 as the location where the prograde and retrograde sheets smoothly connect. The discriminant's other roots are at $a^{2}=1$ or when

$$
\left(a_{\mathrm{dgn} .}(e)\right)^{2}=\frac{(1-e)(3+e)^{3}}{(1+e)(3-e)^{3}} .
$$

That is, $S_{\text {equat }}(p)$ has degenerate roots when $a=a_{\mathrm{dgn} .}$. Since $S_{\text {equat }}$ has real coefficients, its roots are either real or come in complex-conjugate pairs. For general values of $(a, e)$, there are either 4 real roots or 2 real and a complex conjugate pair. However along $\left(a_{\mathrm{dgn} .}(e), e\right)$, there are 4 real roots but one pair has multiplicity 2 . The root with multiplicity 2 has value

$$
p_{\mathrm{mul}}=\frac{3-2 e-e^{2}}{3-e},
$$

and note that $0 \leq p_{\text {mul }} \leq 1$, with equality occurring at the endpoints in $e$. Since this is less than or equal to 1 , it is always unphysical, except at $(a=1, e=0)$. The number of equatorial roots as a function of $(a, e)$ is summarized in Fig. 4.

Since $p_{\text {mul }}$ appears at an unphysically small value of $p$, we can safely ignore these extra solutions, and focus on the outermost two, which are, in increasing magnitude, the prograde and retrograde equatorial separatrices $p_{\text {sep }}^{ \pm \text {equat }}(a, e)$, respectively. Again because of monotonicity, we can bracket the location of these roots by looking at the Schwarzschild and extremal limits. As before for $a=0, p_{\mathrm{sep}}=6+2 e$. The $x= \pm 1, a=1$ polynomial was previously given in factorized form in Eq. (24). The roots are all real, and they are (ordered by value)

$$
\begin{aligned}
& p=5+e-4 \sqrt{1+e}, \\
& p=1+e \quad(\text { twice }), \\
& p=5+e+4 \sqrt{1+e} .
\end{aligned}
$$

The smallest of these is less than 1 and hence one of the unphysical solutions. The largest of these is the retrograde, equatorial, extremal separatrix. The intermediate value is the prograde, equatorial, extremal separatrix [13], and one of the unphysical roots has degenerated with this physical one.

These extremal $(a=1)$ values of the separatrix give us brackets for general values of the equatorial separatrix,

$$
\begin{aligned}
1+e & \leq p_{\mathrm{sep}}^{+ \text {equat }}(a, e) \leq 6+2 e, \\
6+2 e & \leq p_{\mathrm{sep}}^{- \text {equat }}(a, e) \leq 5+e+4 \sqrt{1+e} .
\end{aligned}
$$




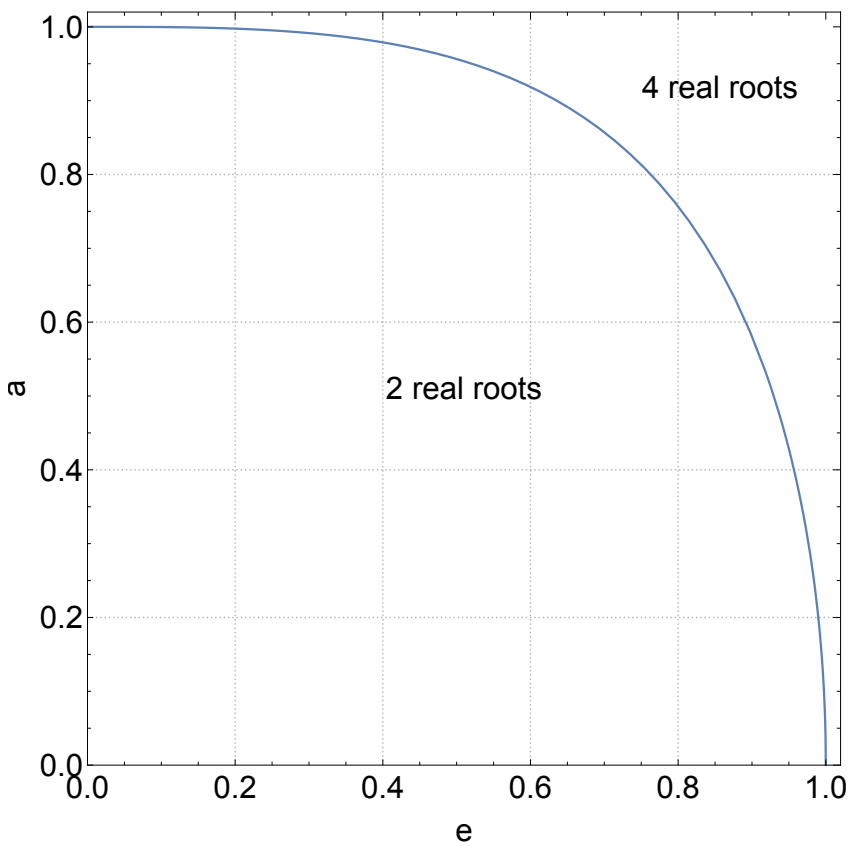

FIG. 4. Number of real roots of the equatorial separatrix polynomial $S_{\text {equat }}(p)$. Along the curve $a_{\text {dgn. }}$ (e) given by Eq. (29), there are 4 real roots but only 3 distinct values, as one pair of complex conjugate roots have degenerated and become real.

\section{Polar}

Polar orbits have $x=0, \sin \iota=1$, which corresponds to $\mathcal{L}_{z}=0$. Recall that the full separatrix polynomial $S(p, e, x)$ is only a function of $x^{2}$ and thus even in $x$. We also know that the function $p_{\text {sep }}(x)$ is monotonic in $x$, with $p_{\text {sep }}$ being smaller for prograde (positive) and larger for retrograde (negative) values of $x$. Therefore as $x$ goes through 0 , the physically relevant sheet of the solution set is crossing through the polar value $p_{\text {sep }}^{\text {pol }}$, in a simple root. But since $S$ depends only on $x^{2}$, the unphysical sheet is simply the reflection of the physical one with $x \rightarrow-x$. Thus there is a degeneracy at $x=0$, and further the polynomial factors as a square of a sextic. That is,

$$
S(x=0)=S_{\mathrm{pol}}(a, p, e)^{2},
$$

where the sextic is

$$
\begin{aligned}
& S_{\text {pol }}(a, p, e)=p^{5}(-6-2 e+p) \\
& \quad+a^{2} p^{3}\left(-4[-1+e][1+e]^{2}+[3+e(2+3 e)] p\right) \\
& \quad-a^{4}(1+e)^{2} p\left(6+2 e^{3}+2 e[-1+p]-3 p-3 e^{2}[2+p]\right) \\
& \quad+a^{6}(-1+e)^{2}(1+e)^{4} .
\end{aligned}
$$

From monotonicity, the minima and maxima of the polar separatrix occur respectively at $(a=1, e=0)$ and $(a=0, e=1)$. That is, for arbitrary $a$ and $e$, the polar separatrix always lies in this interval,

$$
p_{\mathrm{sep}}^{\mathrm{pol}}(1,0) \leq p_{\mathrm{sep}}^{\mathrm{pol}}(a, e) \leq p_{\mathrm{sep}}^{\mathrm{pol}}(0,1) ，
$$

with equality only at the appropriate corners of the unit square in $(a, e)$ space.

When $a=0$, we have to recover Eq. (21), and indeed here we get $S_{\text {pol }}(a=0)=p^{5}(p-6-2 e)$, again leading to $p_{\mathrm{sep}}^{\mathrm{Schw}}=6+2 e$. Thus the global maximum for the polar separatrix is $p_{\text {sep }}^{\text {pol }}(0,1)=8$.

For the extremal limit $a=1$ there is no major simplification. But at the endpoints $e=0$ and $e=1$ there is, respectively,

$$
\begin{aligned}
& S_{\text {pol }}(a=1, e=0)=(-1+p)^{2}\left(1-4 p-6 p^{2}-4 p^{3}+p^{4}\right) \\
& S_{\text {pol }}(a=1, e=1)=p^{2}\left(16+8 p^{2}-8 p^{3}+p^{4}\right) .
\end{aligned}
$$

From these we can find the real nontrivial roots of the separatrix polynomial at these corners of the unit square,

$$
\begin{aligned}
p_{\mathrm{sep}}^{\mathrm{pol}}(a=1, e=0)=1 & +\sqrt{3}+\sqrt{3+2 \sqrt{3}}, \\
\approx 5.27, & \\
p_{\mathrm{sep}}^{\mathrm{pol}}(a=1, e=1)=\frac{2}{3} & \left(3+(54-6 \sqrt{33})^{1 / 3}\right. \\
& \left.+(6(9+\sqrt{33}))^{1 / 3}\right),
\end{aligned}
$$

$$
\approx 6.77 \text {. }
$$

Thus the global minimum for the polar separatrix is $p_{\text {sep }}^{\text {pol }}(1,0) \approx 5.27$.

\section{Circular and spherical orbits}

Specializing to spherical orbits entails setting $e=0$. Unfortunately the separatrix polynomial does not factor further at $e=0$. This polynomial, $S(e=0)$, agrees with one previously presented in Appendix A of Ref. [25], based on the homoclinic orbit approach of Ref. [21].

Of course we can take further special cases where it does factor. For example, the equatorial circular separatrix polynomial is

$S(e=0, x=1)=p^{8}\left(9 a^{4}+(-6+p)^{2} p^{2}-2 a^{2} p(14+3 p)\right)$.

The quartic can be solved by radicals, and the two physical solutions (prograde and retrograde) are the classic result of the equatorial ISCO given by Bardeen, Press, and Teukolsky [20].

Another special case of interest is spherical orbits around an extremal black hole. Here we get the factorization

$$
S(e=0, a=1)=(p-1)^{3} S_{\text {sph., ext. }}(p, x),
$$




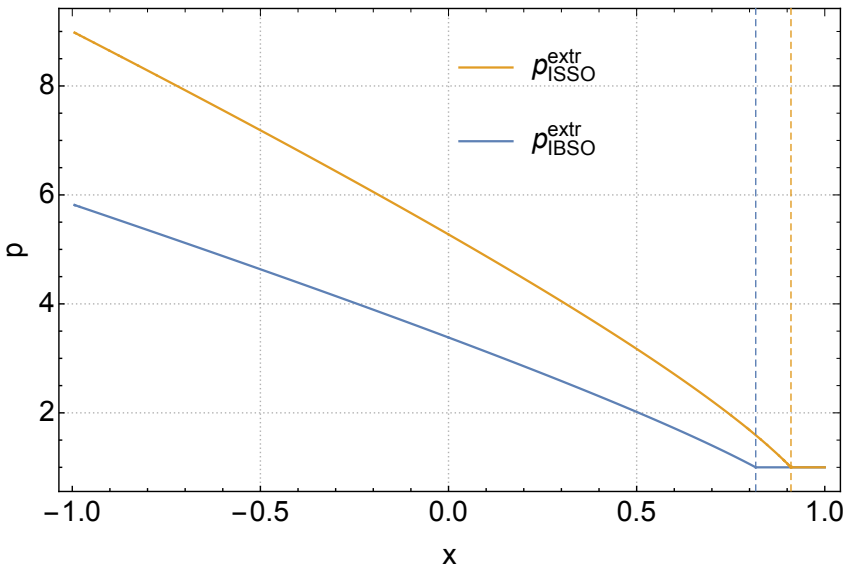

FIG. 5. Location of the innermost stable spherical orbit (blue, solid curve) and the innermost bound spherical orbit (yellow, solid curve) about an extremal $(a=1)$ black hole. Each curve has a kink at a different inclination $x$ (marked by the vertical, dashed lines), where two roots of the associated polynomials cross each other linearly, as discussed in Secs. IV D and C 4.

where the ninth degree factor is

$$
\begin{aligned}
S_{\text {sph., ext. }}= & p^{9}-9 p^{8}+12 p^{7} z_{m}^{2} \\
& +36 p^{6} z_{m}^{2}+30 p^{5} z_{m}^{4} \\
& -30 p^{4} z_{m}^{4}-36 p^{3} z_{m}^{6} \\
& -12 p^{2} z_{m}^{6}+9 p z_{m}^{8}-z_{m}^{8},
\end{aligned}
$$

where $z_{m}^{2}=1-x^{2}$. This polynomial may be described as "anti-reciprocal" in two indeterminates, in the sense that $S_{\text {sph., ext. }}\left(p, z_{m}\right)=-p^{9} z_{m}^{8} S_{\text {sph., ext. }}\left(p^{-1}, z_{m}^{-1}\right)$.

Here we see an interesting phenomenon. For most values of $x$, one root that varies smoothly with $x$ is the physical root $p_{\text {sep }}^{\text {sph. ext. }}(x)$. But, this root crosses $p=1$ linearly at some critical value $x_{\text {kink }}^{\text {sph. ext. }}$. Above this value of $x$, the root $p=1$ becomes the physically relevant root, and this leads to a kink in the graph of $p_{\mathrm{sep}}^{\text {sph. ext. }}(x)$. We find the inclination where the nonic also has a root at $p=1$ by setting $p$ to 1 , leaving us to solve the polynomial $0=8 x^{2}\left(x^{2}-2\right)\left(x^{4}+4 x^{2}-4\right)$. We find the location of the kink is

$$
x_{\text {kink }}^{\text {sph. ext. }}=\sqrt{2(\sqrt{2}-1)} .
$$

Ref. [26] independently derived this result at the same time as this work. From Eq. (46) when $a=1$ and $x \geq$ $x_{\text {kink }}^{\text {sph. ext. }}$, the ISSO is at $p=1$. This kink behavior can be seen in Fig. 5 .

\section{E. Parabolic trajectories}

Parabolic encounters are astrophysically interesting for modeling tidal disruptions of ordinary stars around supermassive black holes [27-29]. They are also interesting as potential sources of gravitational wave bursts [30,31]. A parabolic encounter has $e=1$, which sends the apocenter $r_{1}$ to infinity while $r_{2}=p / 2$ remains finite. Notice that sending one root of $R(r)$ to infinity depresses the quartic to a cubic, which happens when $\mathcal{E}=1$.

Parabolic trajectories are technically not bound orbits. They are another type of parameter space separatrix, between eccentric (bound) and hyperbolic (unbound) trajectories. The set of all parabolic orbits also connects to the separatrix between bound and plunging orbits which we are analyzing in this paper, simply by restricting to $e=1$ in $S(a, p, e, x)$. This reflects an interesting phase space geometry which is beyond the scope of this work.

The separatrix polynomial also factorizes at $e=1$,

$$
S(e=1)=p^{4} S_{\text {para }}(a, p, x),
$$

where the nontrivial factor is

$$
\begin{aligned}
S_{\text {para }}(a, p, x)= & 16 a^{4}\left[16 a^{4}+24 a^{2} p^{2}+p^{3}(9 p-32)\right] x^{4} \\
- & 8 a^{2}\left[64 a^{6}+80 a^{4} p^{2}+p^{5}(3 p-8)\right. \\
& \left.+4 a^{2} p^{3}(7 p-24)\right] x^{2} \\
+ & {\left[16 a^{4}+8 a^{2} p^{2}+(p-8) p^{3}\right]^{2} }
\end{aligned}
$$

Besides the $p$ degree being lowered, $S_{\text {para }}$ is only quadratic in $x^{2}$, meaning it is straightforward to give an explicit parametric description of the surface. Before doing so, we will further specialize to equatorial parabolic encounters to find the $p$ extrema of this slice through the separatrix. Setting $x=1$, we have the further simplification

$$
\begin{aligned}
& S_{\text {para }}(a, p, x=1)=p^{4}\left(4 a^{2}-8 p-4 a p+p^{2}\right) \\
& \times\left(4 a^{2}-8 p+4 a p+p^{2}\right) .
\end{aligned}
$$

Besides the unphysical roots at $p=0$, there are two additional quadratic factors which can be solved directly. The roots of physical interest give the extrema of the parabolic separatrix as a function of $a$,

$$
p_{\text {para }}^{ \pm}(a)=2(2+2 \sqrt{1 \pm a} \pm a) .
$$

The plus signs are taken for retrograde (larger $p$ ), and the minus signs are taken for prograde (smaller $p$ ).

To find the parametric description of the parabolic separatrix, match coefficients $(A, B, C)$ in $S_{\text {para }}=A x^{4}+$ $B x^{2}+C$, then solve for $x^{2}$ in $0=S_{\text {para }}$,

$$
x_{\text {para }}^{2}(a, p)=\frac{-B \pm \sqrt{D}}{2 A},
$$

where the discriminant is $D=2^{13} a^{4} p^{7}\left[4 a^{2}+p(p-4)\right]^{2}$. If one takes the upper sign in Eq. (51), the values of $x_{\text {para }}^{2}$ are always greater than 1 and thus unphysical; therefore take the minus sign in Eq. (51). The parameter $p$ lies in the domain $p_{\text {para }}^{-}(a) \leq p \leq p_{\text {para }}^{+}(a)$, and the image $x_{\text {para }}^{2}(a, p)$ covers $[0,1]$ on this domain. 


\section{NUMERICAL IMPLEMENTATION}

In this section we assume that numerical values are given for $0 \leq a \leq 1,0 \leq e \leq 1$, and $-1 \leq x \leq+1$. Then $S(a, p, e, x)$ is a univariate $12^{\text {th }}$ degree polynomial in $p$ with real coefficients, our goal is to find certain real roots.

Specifically, given values for $a$ and $e_{\text {sep }}, x_{\text {sep }}$ we present a method to rapidly compute $p_{\text {sep }}$. Before we present our new method based upon numerically finding the roots of the separatrix polynomial we review the previous methods in the literature for numerically computing the separatrix.

\section{A. Earlier approaches}

Given a set of geodesic parameters, determining if an orbit is stable is straightforward. Stable orbits have $r_{2}-$ $r_{3}>0$, when both are real, and these roots are easily evaluated as follows. First, $\left(\mathcal{E}, \mathcal{L}_{z}, \mathcal{Q}\right)$ can be expressed explicitly in terms of $(p, e, x)$ by following the procedure detailed in Appendix B of Schmidt [19]. These analytic expressions involve nested radicals, and thus may become complex. Next we follow Fujita and Hikida [32] to find $r_{3}$ and $r_{4}$. Depress the quartic $R(r)$ by the known quadratic factor $\left(1-\mathcal{E}^{2}\right)\left(r_{1}-r\right)\left(r-r_{2}\right)$. This leaves the quadratic $\left(r-r_{3}\right)\left(r-r_{4}\right)$ which is solved explicitly for $r_{3}$ and $r_{4}$, again in terms of nested radicals, so $r_{3}$ and $r_{4}$ may also become complex.

Finding the precise location of the separatrix in a robust and computationally efficient way is more challenging. This is because, as mentioned above, in some regions of the parameter space $r_{2}-r_{3}$ becomes complex. This causes, e.g., a Newton-Raphson root finding scheme to fail - see Fig. 6 and, e.g., Appendix A of [9]. These challenging regions of the parameter space occur for high spin, near prograde equatorial orbits.

The problems mentioned above occur when the root finder steps over the separatrix to a value of $p<p_{\text {sep }}$ and the algorithm is unsure how to proceed because the function it is evaluating has become complex. One way to avoid this is to use a bisection-like method to seek the root strictly from above. In this method you pick an initial value of $p$ large enough to ensure $p>p_{\text {sep }}$, check if $r_{2}>r_{3}$ and if so decrease $p$ by some small amount $\Delta p_{\text {sep. }}$. This is repeated until $r_{3}$ becomes complex at which point the previous value of $p$ is returned to and now $\Delta p_{\text {sep }}$ is halved and the process repeats. As this is a bisection-like method it is robust but does not converge quickly.

A robust approach that can use rapidly convergent numerical root finding was introduced into the Black Hole Perturbation Toolkit [14] in 2018 by one of us. This used an extension of the method of Ref. [21] from equatorial to generic orbits. We give the equations for this extension in Appendix D. Recently Ref. [33] published equations for a similar extension. In this approach one picks values for $e_{\text {sep }}, x_{\text {sep }}$ and then root finds for an unstable circular orbit radius, $r_{u}$, in the range $r_{\text {ibso }} \leq r_{u}$. With $r_{u}$ strictly bounded below the method is robust

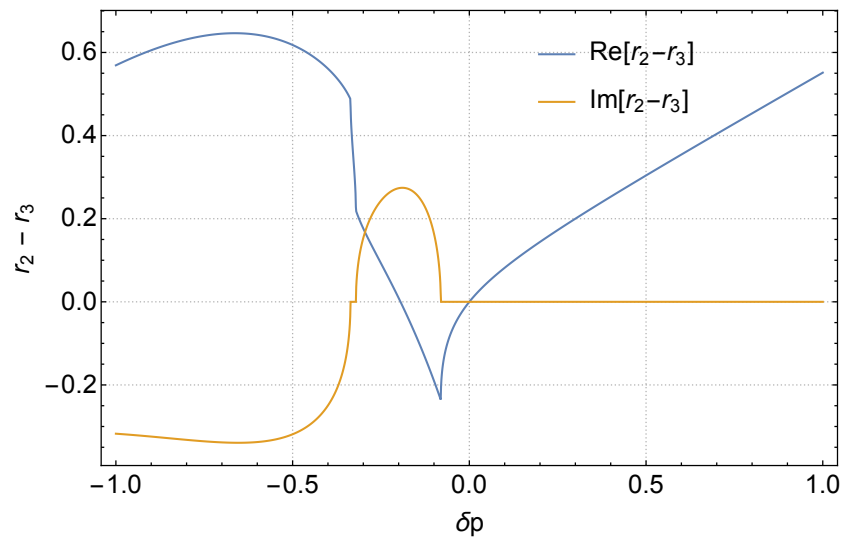

FIG. 6. The difference $r_{2}-r_{3}$ plotted as a function of $\delta p=$ $p-p_{\text {sep }}$ for $a=0.998, e=0.9, x=0.95$. The plot shows the real and imaginary parts in blue and yellow, respectively. The separatrix is at $\delta p=0$ which corresponds to $p_{\text {sep }}=2.10085$ where $r_{2}-r_{3}=0$. The non-smoothness of this function makes it difficult to numerically root find on $r_{2}-r_{3}$ to find the separatrix.

and rapidly convergent numerical root finding techniques, like a Newton-Raphson method, can be employed. The downside to this method is that first $r_{\text {ibso }}$ must be found and this adds to the computational overhead.

We now discuss new approaches which are faster and guaranteed to find $p_{\text {sep }}$.

\section{B. Global root-finding}

From the fundamental theorem of algebra, $S$ has 12 complex roots for $p$, and several methods exist to find all roots simultaneously. One standard "black-box" approach [34] is the method of Jenkins and Traub. There exists both a general version for polynomials with complex coefficients, and a more adapted algorithm for polynomials with real coefficients [35].

Another popular algorithm is the Aberth method [36], which converges cubically to simple roots, but only exists in a complex form. If one were to initiate the Aberth method with purely real guesses for a real polynomial, the iteration scheme would never push the guesses into the complex plane, and would thus fail to find complex roots. Therefore it is important to start with complex guesses, though this means that all roots will acquire some imaginary part, even if the root is exactly on the real axis. Therefore using the Aberth method (or the complex version of the Jenkins-Traub method) requires testing roots $p_{i}$ for realness via $\left|\operatorname{Im}\left[p_{i}\right]\right| \leq \epsilon$ with some arbitrary choice of $\epsilon>0$.

Such black-box global root-finding methods are implemented in most computer algebra systems and numerical libraries. For example, in MAтнематіCA, the command NRoots [] implements both the Jenkins-Traub and Aberth methods. 
For special values such as $x=0, x^{2}=1$, or $e=0$, one should use the appropriate simplified polynomial. Here we give the generic algorithm, for general points:

1. Find all complex roots $p_{i}$, and select the real roots as those that satisfy $\left|\operatorname{Im}\left[p_{i}\right]\right| \leq \epsilon$ with some $\epsilon>0$ determined by the required precision.

2. If $x<0$, the orbit is retrograde and thus the desired root is the largest real root.

3. If $x>0$, the orbit is prograde and thus the desired root is the second largest real root.

\section{Real root isolation}

For polynomials with real coefficients, it is possible to bound the number of real roots and to isolate each real root into an interval of the real line, with black-box "real-root isolation" algorithms. At their most basic, these algorithms arise from Descartes' rule of signs [37], with improvement due to Sturm's theorem [38]. Using real-root isolation, one is guaranteed to find brackets for all the simple real roots of $S(p)$ automatically. Such algorithms are implemented in several computer algebra systems, for example in the SAGE module sage.rings.polynomial.real_roots, or in the MATHEMATICA commands RootIntervals [] or NSolve [. . , Reals].

For special values such as $x=0, x^{2}=1$, or $e=0$, the separatrix polynomial is not square-free, and one should instead focus on solving the nontrivial factor such as $S_{\mathrm{pol}}$. Here we give the generic algorithm, for general points:

1. Find isolating intervals for all roots of $S(p)$ in the admissible physical range, $1 \leq p \leq 6+4 \sqrt{2} \approx 11.66$.

2. If $x<0$, the orbit is retrograde and thus the desired root is the largest real root.

3. If $x>0$, the orbit is prograde and thus the desired root is the second largest real root.

\section{Bracketing of roots}

Rather than relying on a black box algorithm to find isolating intervals for real roots, we can analytically find them, using all the limiting cases presented in Sec. IV. The method we describe here is also the fastest and most robust, and the one that is implemented in the Black Hole Perturbation Toolkit [14].

First note that we have the brackets,

$$
\begin{aligned}
p_{\mathrm{sep}}^{+ \text {equat }}(a, e) & \leq p_{\mathrm{sep}}^{\mathrm{pro}}(a, e, x) \leq p_{\mathrm{sep}}^{\mathrm{pol}}(a, e), \\
p_{\mathrm{sep}}^{\mathrm{pol}}(a, e) & \leq p_{\mathrm{sep}}^{\mathrm{ret}}(a, e, x) \leq p_{\mathrm{sep}}^{- \text {equat }}(a, e),
\end{aligned}
$$

where \pm equat refer to the prograde/retrograde equatorial orbits. These serve as brackets, if we know the values of the polar and equatorial separatrices. Those values are found via their own bracketed root-finding. The algorithm proceeds as follows, given some inputs $a, e, x$ :

1. In all cases one needs to find $p_{\mathrm{sep}}^{\mathrm{pol}}(a, e)$, by polishing the single simple root of the sextic $S_{\text {pol }}(p)$ given in Eq. (37), within the bracket

$$
1+\sqrt{3}+\sqrt{3+2 \sqrt{3}} \leq p_{\mathrm{sep}}^{\mathrm{pol}}(a, e) \leq 8 .
$$

2. If $x=0$, the orbit is polar and the separatrix has been found. Otherwise:

(a) If $x>0$, find $p_{\text {sep }}^{+ \text {equat }}(a, e)$, by polishing the single simple root of Eq. (23) within the bracket

$$
1+e \leq p_{\text {sep }}^{\text {equat }}(a, e) \leq 6+2 e .
$$

Now with $p_{\text {sep }}^{+ \text {equat }}(a, e)$ and $p_{\text {sep }}^{\mathrm{pol}}(a, e)$ in hand, polish the single simple root of the full $12^{\text {th }}$ degree $S(p)$ within the bracket Eq. (52).

(b) If $x<0$, one can omit finding $p_{\text {sep }}^{\text {-equat }}(a, e)$, since there is only ever one root of the separatrix polynomial between $p_{\mathrm{sep}}^{\mathrm{pol}}(a, e)$ and the maximum possible value of the separatrix (discussed in Sec. IV B), $p_{\text {sep }}^{\max }=6+4 \sqrt{2} \approx 11.66$. Therefore polish the single simple root of the full $12^{\text {th }}$ degree $S(p)$ within the bracket $p_{\mathrm{sep}}^{\mathrm{pol}}(a, e) \leq p_{\mathrm{sep}}(a, e, x) \leq 12$.

\section{E. Implementation in the Black Hole Perturbation Toolkit}

The algorithm presented above to compute the separatrix is implemented in the KerrGeodesics MATHEMATICA package of the Black Hole Perturbation Toolkit [14]. It can be accessed with a function called KerrGeoSeparatrix $[a, e, x]$. This algorithm replaced the slower algorithm outlined in Appendix D. The new method takes $\sim 1 \mathrm{~ms}$ to calculate the location of the separatrix to machine precision. ${ }^{1}$ This is roughly 45 times faster than the previous implementation which relied on multiple root finding steps.

In addition to numerically finding $p_{\text {sep }}$ the KerrGeoSeparatrix $[a, e, x]$ function will also return the closed form analytic results for the special cases presented in Sec. IV.

\section{SUMMARY AND FUTURE WORK}

In this article we have examined the separatrix between stable bound orbits and plunging orbits for test body

\footnotetext{
${ }^{1}$ This is measured on a $2.5 \mathrm{GHz}$ Macbook Pro laptop using MATHEMATICA 12 .
} 
motion in Kerr spacetime. We found the generic polynomial whose roots are the location of the separatrix in the $(p, e, x)$ parameter space - stated in Eq. (20), with coefficients tabulated in Appendix A and in the supplementary MATHEMATICA notebook [24]. For generic orbits the polynomial is $12^{\text {th }}$ degree in $p$ and $4^{\text {th }}$ degree in $x^{2}$ so in this case closed form solutions are either not available or practical. For these orbits we provide robust algorithm for numerically finding separatrix in Sec. V and provided an example implementation in the Black Hole Perturbation Toolkit [14]. For special classes of orbits the separatrix polynomial simplifies and we can find analytic solutions. These results are presented in Sec. IV. In Appendix C we also consider the special $\mathcal{E}=1$ case of the innermost bound spherical orbits (IBSO).

We have focused on bound geodesic motion in the Kerr spacetime in this work. Generalizations and extensions are possible. We expect that in the Kerr-Newman spacetime [and perhaps even the Kerr-Newman-Taub-NUT(anti-)de Sitter family], the separatrix is also an algebraic variety and can be reduced to a single polynomial in parameter space. In a more astrophysically relevant extension, it would also be interesting to consider the case where the orbiting test body is spinning. In this case the body's spin couples to the local curvature of the spacetime [39-41] and this modifies the orbital motion [42]. This in turn modifies the location of the separatrix. To the best of our knowledge, the change to the separatrix due the spin on the test body has only been studied in the circular, equatorial orbit case [43].

\section{ACKNOWLEDGEMENTS}

LCS acknowledges Daniel McLaury for helpful discussion. NW gratefully acknowledges support from a Royal Society - Science Foundation Ireland University Research Fellowship.

\section{Appendix A: Coefficients of $S$}

This section contains the coefficients $S_{n}$ in the expansion of the separatrix polynomial Eq. (20), repeated here for convenience,

$$
S(a, p, e, x)=\sum_{n=0}^{12} S_{n} p^{n} .
$$

As mentioned earlier, it is possible to develop the separatrix polynomial with the angular parameter being any of $x, z_{m}, \sin \iota$, or $\cos \iota$. The relationships $x^{2}+z_{m}^{2}=1$ and $\sin ^{2} \iota+\cos ^{2} \iota=1$ allow converting between pairs of them, so we present coefficients in two angular parameterizations below. We have also provided a MATHEMATICA notebook as a machine-readable supplement to this article, containing the derivation and resulting polynomial [24].

\section{As a function of $x$}

$$
\begin{aligned}
S_{12}= & 1 \\
S_{11}= & -4(3+e) \\
S_{10}= & 4(3+e)^{2}+2 a^{2}\left(3+2 e+3 e^{2}-2[3+e(2+e)] x^{2}\right) \\
S_{9}= & 4 a^{2}\left[-7+e\left(-7+e\left[-13-5 e+4(3+e) x^{2}\right]\right)\right] \\
S_{8}= & -16 a^{2}(-1+e)(1+e)^{2}(3+e)\left(-1+x^{2}\right)+a^{4}\left(15+20 e+26 e^{2}+20 e^{3}+15 e^{4}-4[9+e(12+e[18+e(12+5 e)])] x^{2}\right. \\
& \left.+2[15+e(2+e)(10+3 e[2+e])] x^{4}\right) \\
S_{7}= & -8 a^{4}(1+e)^{2}(-1+x)(1+x)\left(-3+e-e^{2}-5 e^{3}+[15+e(-5+3 e[1+e])] x^{2}\right) \\
S_{6}= & -4 a^{4}(1+e)^{2}(-1+x)(1+x)\left(-2\left[11-14 e^{2}+3 e^{4}\right]\left[-1+x^{2}\right]\right. \\
& \left.+a^{2}\left[5+6 e^{2}+5 e^{4}-\left(5+e^{2}[6+e(8+5 e)]\right) x^{2}+(-1+e)(3+e)(3+e[2+e]) x^{4}\right]\right) \\
S_{5}= & 8 a^{6}(-1+e)(1+e)^{3}\left(-1+x^{2}\right)^{2}\left(3+e+e^{2}-5 e^{3}+2\left[6+e\left(2+e+e^{2}\right)\right] x^{2}\right) \\
S_{4}= & a^{6}(1+e)^{4}\left(-1+x^{2}\right)^{2}\left(-16[-3+e][-1+e]^{2}[1+e]\left[-1+x^{2}\right]\right. \\
& \left.+a^{2}\left[15+e(-20+e[26+5 e(-4+3 e)])+6 x^{2}-2 e(2+e)(2+e[-6+5 e]) x^{2}+(-1+e)^{2}(3+e)^{2} x^{4}\right]\right) \\
S_{3}= & -4 a^{8}(-1+e)(1+e)^{5}\left(-1+x^{2}\right)^{3}\left(7-7 e+13 e^{2}-5 e^{3}+[-1+e]\left[7+e^{2}\right] x^{2}\right) \\
S_{2}= & 2 a^{8}(-1+e)^{2}(1+e)^{6}\left(-1+x^{2}\right)^{3}\left(2[-3+e]^{2}\left[-1+x^{2}\right]+a^{2}\left[-3+2 e-3 e^{2}+(-1+e)(3+e) x^{2}\right]\right) \\
S_{1}= & -4 a^{10}(-3+e)(-1+e)^{3}(1+e)^{7}\left(-1+x^{2}\right)^{4} \\
S_{0}= & a^{12}(-1+e)^{4}(1+e)^{8}\left(-1+x^{2}\right)^{4}
\end{aligned}
$$




\section{As a function of $\sin \iota$}

Here we use the shorthand $s=\sin \iota$.

$$
\begin{aligned}
S_{12} & =1 \\
S_{11} & =-4(3+e) \\
S_{10} & =4(3+e)^{2}+2 a^{2}\left(-3-2 e+e^{2}+2[3+e(2+e)] s^{2}\right) \\
S_{9} & =-4 a^{2}\left(7+e\left[7+e+e^{2}+4 e(3+e) s^{2}\right]\right) \\
S_{8} & =16 a^{2}(-1+e)(1+e)^{2}(3+e) s^{2}+a^{4}\left[(-3+e)^{2}(1+e)^{2}+2(1+e)(-15+e[-5+e(-1+5 e)]) s^{2}+4[3+e(2+e)]^{2} s^{4}\right] \\
S_{7} & =8 a^{4}(1+e)^{2} s^{2}\left(15-5 e+e^{2}-3 e^{3}-2\left[9+e\left(-3+e+e^{2}\right)\right] s^{2}\right) \\
S_{6} & =4 a^{4}(1+e)^{2} s^{2}\left(2\left[-1+e^{2}\right]\left[7+e^{2}+2\left(-9+e^{2}\right) s^{2}\right]+a^{2}\left[-4+9 s^{2}+e^{2}\left(-2+2[-2+e] e+[8+e(4+3 e)] s^{2}\right)\right]\right) \\
S_{5} & =-8 a^{6}(-1+e)(1+e)^{3} s^{2}\left(3+e-e^{2}+e^{3}+2\left[-3-e+2 e^{3}\right] s^{2}\right) \\
S_{4} & =a^{6}(1+e)^{4} s^{2}\left(16[-3+e][-1+e]^{2}[1+e] s^{2}+a^{2}\left[2(-3+e)(-1+e)^{2}(1+e)+(21+e[-28+e(22+e[-12+13 e])]) s^{2}\right]\right) \\
S_{3} & =-4 a^{8}(-1+e)(1+e)^{5}(-7+e[7+e(-13+5 e)]) s^{4} \\
S_{2} & =2 a^{8}(-1+e)^{2}(1+e)^{6}\left(2[-3+e]^{2}+a^{2}[3+e(-2+3 e)]\right) s^{4} \\
S_{1} & =-4 a^{10}(-3+e)(-1+e)^{3}(1+e)^{7} s^{4} \\
S_{0} & =a^{12}(-1+e)^{4}(1+e)^{8} s^{4}
\end{aligned}
$$

\section{Appendix B: Series in spin $a$}

Deriving a series solution for $p_{\text {sep }}(a, e, x)$ is very straightforward since we have the explicit polynomial $S(a, p, e, x)$. The only difficulty is that while $S$ has multiple solutions, our series must be able to pick an individual "leaf." This means we have to make a branch choice at some point.

At $a=0$, we saw in Sec. IV A that the separatrix is given by $p_{\mathrm{sep}}^{\mathrm{Schw}}=6+2 e$. Now we pose the ansatz

$$
p_{\text {sep }}=\sum_{k=0}^{\infty} p_{k}(e, x) a^{k}
$$

where $p_{0}(e, x)=6+2 e$. This ansatz can be inserted into the polynomial $S$ and solved order-by-order in $a$. At linear order there are two possible solutions, as mentioned before, and we have to make a branch choice to pick the physical leaf. If we truncate at order $a^{2}$, we have to solve

$$
0=1024 a^{2}(3+e)^{9}\left[(3+e) p_{1}^{2}-32(1+e) x^{2}\right]+\mathcal{O}\left(a^{3}\right) .
$$

The two solutions for $p_{1}$ are the prograde and retrograde leaves, which have coalesced in the $a \rightarrow 0$ limit. We need $p_{\text {sep }}$ to decrease with increasing $x$, so we choose the sign

$$
p_{1}(e, x)=-x \sqrt{\frac{32(1+e)}{3+e}} .
$$

This first term was previously found for the equatorial case in [13]. After this sign has been fixed, all higher terms $p_{k}$ come from solving a linear equation by truncating at order $a^{k+1}$. The first few of these are

$$
\begin{gathered}
p_{2}=\frac{-11-e^{3}+4 x^{2}+e\left(-11+4 x^{2}\right)+e^{2}\left(-9+8 x^{2}\right)}{2(3+e)^{2}} \\
p_{3}=\frac{(1+e)^{1 / 2} x}{\sqrt{2}(3+e)^{7 / 2}}(-4[7+e(7+6 e)] \\
\left.\quad+3(5+e[5-(-7+e) e]) x^{2}\right)
\end{gathered}
$$

and so on up to arbitrary order. In the companion MATHEMATICA notebook [24], we provide these coefficients up through and including $p_{6}$.

Unfortunately, this series is not very useful at high spin. If we keep terms up to $a^{6}$, the maximum error in $p$ across $(e, x)$ is $\sim 1 \%$ when $a=0.8$. But by a spin of $a=0.95$, the maximum error is already $\sim 10 \%$. Therefore we do not recommend the series approach.

\section{Appendix C: Innermost bound spherical orbits}

The location of the innermost bound spherical orbits (IBSOs) can be found following the same approach as that for finding the separatrix polynomial. Bound orbits have $\mathcal{E}<1$, and the limit $\mathcal{E} \rightarrow 1$ gives marginally bound orbits. Note that marginally bound spherical orbits are not stable, being interior to the innermost stable circular orbits [20]. They are interesting nonetheless so we demonstrate how to find the IBSO polynomial.

We can again form a polynomial system to define the location of the IBSO. By setting $\mathcal{E}=1$, we depress the quartic to a cubic, but we will still number the remaining real roots as $r_{1} \geq r_{2} \geq r_{3}$. Being a spherical orbit, 
$r_{1}=r_{2}=p$ is a double root. Further we are not interested in stability, only the existence of the spherical orbit with $\mathcal{E}=1$. Thus our system is

$$
\left\{\begin{array}{l}
0=x^{2} \Theta(x ; \mathcal{E}=1) \\
0=R(p ; \mathcal{E}=1) \\
0=R^{\prime}(p ; \mathcal{E}=1) .
\end{array}\right.
$$

The last two equations implement the condition that $p$ is a double root and hence spherical. This system is polynomial in $\left(a, p, x, \mathcal{L}_{z}, \mathcal{Q}\right)$. We can again eliminate $\mathcal{L}_{z}, \mathcal{Q}$ with a computer algebra system leaving a single polynomial in $(a, p, x)$. After removing some unimportant prefactors, we have the IBSO polynomial,

$$
\begin{aligned}
I= & (-4+p)^{2} p^{6}+2 a^{2} p^{5}\left(-8+2 p+4 x^{2}-3 p x^{2}\right) \\
& +a^{4} p^{3}\left[-8\left(1-3 x^{2}+2 x^{4}\right)+p\left(6-14 x^{2}+9 x^{4}\right)\right] \\
& +2 a^{6} p^{2}\left(2-5 x^{2}+3 x^{4}\right)+a^{8}\left(-1+x^{2}\right)^{2} .
\end{aligned}
$$

As with the separatrix polynomial, we can take limits and get simplifications.

\section{Schwarzschild IBSO}

Setting $a=0$ we get the factorization,

$$
I(a=0)=p^{6}(p-4)^{2},
$$

recovering the Schwarzschild IBSO at $p=4$.

\section{Equatorial IBSO}

Setting $x=1$ we get the factorization,

$I(x=1)=p^{4}\left(p^{2}-4 p-2 a p+a^{2}\right)\left(p^{2}-4 p+2 a p+a^{2}\right)$.

The quadratic factors can be solved by radicals. Two of the roots are in the physical region, recovering the classical result from [20],

$$
p_{\text {IBSO }}^{ \pm \text {equat }}=2 \mp a+2 \sqrt{1 \mp a} .
$$

\section{Polar IBSO}

Setting $x=0$ we get the factorization,

$$
I(x=0)=\left(p^{4}-4 p^{3}+2 a^{2} p^{2}+a^{4}\right)^{2} .
$$

As this is the square of a quartic, there are four solutions by radicals. The physical solution for $p_{\mathrm{IBSO}}^{\mathrm{pol}}$ is

$$
\begin{aligned}
p_{\text {IBSO }}^{\text {pol }} & =1+\sqrt{X_{2}}+\sqrt{3-a^{2}+\frac{2-a^{2}}{\sqrt{X_{2}}}-X_{2}} \\
X_{2} & =1-\frac{a^{2}}{3}+\frac{2 a^{4}}{3 X_{1}^{1 / 3}+\frac{X_{1}^{1 / 3}}{6}} \\
X_{1} & =a^{4}\left(27+\sqrt{27\left(27-16 a^{2}\right)}\right)-8 a^{6},
\end{aligned}
$$

where all radical expressions are real in the physical region.

\section{Extremal IBSO}

In the extremal limit $a \rightarrow 1$, we get the simplification

$$
\begin{aligned}
I(a=1)=(p-1)^{2}( & (p-6) p^{5}+p^{4}+z_{m}^{4} \\
& +2 p^{2}(1+p)(-1+3 p) z_{m}^{2} \\
& \left.+p(2+9 p) z_{m}^{4}\right)
\end{aligned}
$$

where $z_{m}^{2}=1-x^{2}$. Here we see the same type of phenomenon as already discussed in Sec. IV D. For most values of $x$, one smoothly-varying root of the sextic plays the role of $p_{\text {IBSO }}^{\text {ext. }}$ But this root linearly crosses the constant root at $p=1$ at some critical value of $x_{\text {kink }}^{\text {ext. IBSO }}$ We find this value by inserting $p=1$ into the sextic and thus have to solve $0=4-8 x^{2}+3 x^{4}$ to find the inclination of the kink. We find the location of the kink is

$$
x_{\text {kink }}^{\text {ext. IBSO }}=\sqrt{2 / 3},
$$

which was previously found by other methods in [44]. Thus when $a=1$ and $x \geq x_{\text {kink }}^{\text {ext. IBSO }}$, the IBSO is at $p=1$. This kink behavior can be seen in Fig. 5 .

\section{Appendix D: Numerical method for calculating the separatrix via connection to homoclinic orbits}

In this appendix we generalize the approach of Ref. [21] to generic orbits. In general the radial equation has four distinct roots. On the separatrix two of these roots coalesce so that $r_{2}=r_{3}$. Thus we can write the radial equation in the form

$$
R(r)=-\beta\left(r-r_{4}\right)\left(r-r_{2}\right)^{2}\left(r-r_{1}\right),
$$

where recall that $\beta=1-\mathcal{E}^{2}$. Comparing the coefficients of $r^{2}$ and $r^{3}$ in the above with the same coefficients in Eq. (5) and solving simultaneously for $\left\{r_{1}, r_{2}\right\}$ we find

$$
r_{1}=-r_{2}+\frac{1+\sqrt{1-\beta\left(a^{2} \beta+\mathcal{L}_{z}^{2}+\mathcal{Q}+2 r_{2}\left(\beta r_{2}-1\right)\right)}}{\beta} .
$$


We could now substitute $\left\{\mathcal{E}, \mathcal{L}_{z}, \mathcal{Q}\right\}$ with their values for spherical orbits with radius $r_{2}$ and substitute the result into Eq. (14). This would give us a parametric equation for $p_{\text {sep }}\left(r_{0}, x\right)$ and $e_{\text {sep }}\left(r_{0}, x\right)$. Our goal is to find $p_{\text {sep }}(a, e, x)$. To do this we need to numerically root find to get the solutions of $e=e_{\mathrm{sep}}\left(r_{0}, x\right)$. To do this stably across the entire parameter space we need to bracket the root. The value of $e_{\text {sep }}\left(r_{0}\right)$ varies from $e=0$ when $r_{0}=r_{\text {isso }}$ to $e=1$ at $r_{0}=r_{\text {ibso }}$. Unfortunately, at $r_{0}=r_{\text {ibso }}$ the maximum orbital radius, $r_{1}$, diverges. This can be overcome by writing

$$
r_{1}=\frac{r_{1}^{\mathrm{reg}}}{\gamma}
$$

where $r_{1}^{\text {reg }}$ remains finite as $e \rightarrow 1$ and $\gamma \rightarrow 0$ as $e \rightarrow 1$. In formulating the equation for $r_{1}^{\text {reg }}$ we have to be careful to avoid any divisions by $\beta=\left(1-\mathcal{E}^{2}\right)$ as $\mathcal{E} \rightarrow 1$ as $e \rightarrow 1$. This is easily arranged and we find

$r_{1}^{\mathrm{reg}}=2\left(\mathcal{L}_{z}-a \mathcal{E}\right)^{2}+2 \mathcal{Q}$

$+r_{2}^{2}\left(-1+r_{2} \beta+\sqrt{1-\beta\left(\mathcal{L}_{z}^{2}+\mathcal{Q}+a^{2} \beta-2 r_{2}\left(1-r_{2} \beta\right)\right)}\right)$

$\gamma / r_{2}+\beta r_{2}=$

$2-2 \sqrt{1+\beta\left(-a^{2} \beta-\mathcal{L}_{z}^{2}-\mathcal{Q}-2 \beta r_{2}^{2}+2 r_{2}\right)}$.

With these definitions we can define the eccentricity along the separatrix as

$$
\begin{aligned}
e_{\mathrm{sep}}^{\mathrm{reg}} & =\frac{r_{1}^{\mathrm{reg}}-r_{2} \gamma}{r_{1}^{\mathrm{reg}}+r_{2} \gamma} \\
p_{\mathrm{sep}}^{\mathrm{reg}} & =\frac{2 r_{1}^{\mathrm{reg}} r_{2}}{r_{1}^{\mathrm{reg}}+r_{2} \gamma} .
\end{aligned}
$$

In this equation you can directly substitute $e_{\text {sep }}^{\text {reg }}$ and get $e_{\text {sep }}^{\text {reg }}\left(r_{\text {ibso }}\right)=1$.

Putting it all together the algorithm for robustly locating the generic Kerr separatrix is

1. Pick a value for each of $\{a, e, x\}$.

2. Numerically solve $e_{\text {sep }}^{\text {reg }}\left(r_{2}, x\right)=e$ by root finding between $r_{2}=r_{\text {ibso }}$ and $r_{2}=10$ (this is beyond any value the $r_{\text {isso }}$ can take). In evaluating Eq. (D6) use the formula for $\left\{\mathcal{E}, \mathcal{L}_{z}, \mathcal{Q}\right\}$ for spherical orbits with radius $r_{2}$.

3. Compute $p_{\text {sep }}^{\text {reg }}$ using Eq. (D7).

The above algorithm was implemented into the KerrGeodesics MATHEMATICA package in the Black Hole Perturbation Toolkit. Note this algorithm requires the location of the IBSO to be calculated beforehand. The Toolkit implementation found this by first locating the photon sphere radius, $r_{\mathrm{ph}}$, and then root finding on $\mathcal{E}\left(r_{\text {ibso }}\right)=1$ noting that $r_{\text {ibso }}>r_{\text {ph }}$ to bracket the root. This added two root finds to the process which slowed the algorithm down with respect to the new one presented in the main body of this article. The code in the Black Hole Perturbation Toolkit has now been upgraded to use the more efficient method.

Shortly after the above algorithm was implemented in the Black Hole Perturbation Toolkit Ref. [33] published their generalization of the approach in Ref. [21].
[1] J. E. McClintock, R. Narayan, S. W. Davis, L. Gou, A. Kulkarni, J. A. Orosz, R. F. Penna, R. A. Remillard, and J. F. Steiner, General relativity and gravitation. Proceedings, 19th International Conference, GR19, Mexico City, Mexico, July 4-9, 2010, Class. Quant. Grav. 28, 114009 (2011), arXiv:1101.0811 [astro-ph.HE].

[2] S. Babak, J. Gair, A. Sesana, E. Barausse, C. F. Sopuerta, C. P. L. Berry, E. Berti, P. Amaro-Seoane, A. Petiteau, and A. Klein, Phys. Rev. D95, 103012 (2017), arXiv:1703.09722.

[3] C. P. L. Berry, S. A. Hughes, C. F. Sopuerta, A. J. K. Chua, A. Heffernan, K. Holley-Bockelmann, D. P. Mihaylov, M. C. Miller, and A. Sesana, arXiv:1903.03686 [astro-ph.HE].

[4] P. Amaro-Seoane, C. F. Sopuerta, and M. D. Freitag, Mon. Not. Roy. Astron. Soc. 429, 3155 (2013), arXiv:1205.4713 [astro-ph.CO].

[5] S. Babak, H. Fang, J. R. Gair, K. Glampedakis, and S. A. Hughes, Phys. Rev. D75, 024005 (2007), [Erratum: Phys. Rev.D77,04990(2008)], arXiv:gr-qc/0607007 [gr-qc].

[6] A. J. K. Chua, C. J. Moore, and J. R. Gair, Phys. Rev. D96, 044005 (2017), arXiv:1705.04259 [gr-qc].
[7] A. Ori and K. S. Thorne, Phys. Rev. D62, 124022 (2000), arXiv:gr-qc/0003032 [gr-qc].

[8] R. W. O'Shaughnessy, Phys. Rev. D67, 044004 (2003), arXiv:gr-qc/0211023 [gr-qc].

[9] P. A. Sundararajan, Phys. Rev. D77, 124050 (2008), arXiv:0803.4482 [gr-qc].

[10] A. Apte and S. A. Hughes, Phys. Rev. D100, 084031 (2019), arXiv:1901.05901 [gr-qc].

[11] O. Burke, J. R. Gair, and J. Simón, arXiv:1909.12846 [gr-qc].

[12] G. Compère, K. Fransen, and C. Jonas, arXiv:1909.12848 [gr-qc].

[13] K. Glampedakis and D. Kennefick, Phys. Rev. D66, 044002 (2002), arXiv:gr-qc/0203086 [gr-qc].

[14] Black Hole Perturbation Toolkit, (bhptoolkit.org).

[15] Y. Mino, Phys. Rev. D67, 084027 (2003), arXiv:grqc/0302075 [gr-qc].

[16] N. Warburton, L. Barack, and N. Sago, Phys. Rev. D87, 084012 (2013), arXiv:1301.3918 [gr-qc].

[17] C. Cutler, D. Kennefick, and E. Poisson, Phys. Rev. D50, 3816 (1994).

[18] S. A. Hughes, Phys. Rev. D61, 084004 (2000), [Erratum: Phys. Rev.D90,no.10,109904(2014)], arXiv:gr-qc/9910091. 
[19] W. Schmidt, Class. Quant. Grav. 19, 2743 (2002), arXiv:gr-qc/0202090 [gr-qc].

[20] J. M. Bardeen, W. H. Press, and S. A. Teukolsky, Astrophys. J. 178, 347 (1972).

[21] J. Levin and G. Perez-Giz, Phys. Rev. D79, 124013 (2009), arXiv:0811.3814 [gr-qc].

[22] A. L. Dixon, Proceedings of the London Mathematical Society s2-7, 49 (1909).

[23] D. Kapur, T. Saxena, and L. Yang, in Proceedings of the international symposium on Symbolic and algebraic computation (ACM, New York, 1994) pp. 99-107.

[24] See Supplemental Material at http://link.aps.org/ supplemental/10.1103/PhysRevD.101.064007 or https: //arxiv.org/src/1912.07609/anc for a MATHEMATICA notebook which computes the separatrix polynomial and related quantities.

[25] N. Stone, A. Loeb, and E. Berger, Phys. Rev. D87, 084053 (2013), arXiv:1209.4097 [astro-ph.HE].

[26] G. Compère and A. Druart, arXiv:2001.03478 [gr-qc].

[27] C. R. Evans and C. S. Kochanek, Astrophys. J. Lett. 346, L13 (1989).

[28] N. C. Stone, M. Kesden, R. M. Cheng, and S. van Velzen, Gen. Rel. Grav. 51, 30 (2019), arXiv:1801.10180 [astroph.HE].

[29] K. Hayasaki, S. Zhong, S. Li, P. Berczik, and R. Spurzem, Astrophys. J. 855, 129 (2018), arXiv:1802.06798 [astroph.HE].

[30] C. P. L. Berry and J. R. Gair, Mon. Not. Roy. Astron. Soc. 433, 3572 (2013), arXiv:1306.0774 [astro-ph.HE].

[31] S. Hopper and V. Cardoso, Phys. Rev. D97, 044031 (2018), arXiv:1706.02791 [gr-qc].
[32] R. Fujita and W. Hikida, Class. Quant. Grav. 26, 135002 (2009), arXiv:0906.1420 [gr-qc].

[33] P. Rana and A. Mangalam, Class. Quant. Grav. 36, 045009 (2019), arXiv:1901.02730 [gr-qc].

[34] W. H. Press, S. A. Teukolsky, W. T. Vetterling, and B. P. Flannery, Numerical Recipes 3rd Edition: The Art of Scientific Computing, 3rd ed. (Cambridge University Press, New York, NY, USA, 2007).

[35] M. A. Jenkins and J. F. Traub, SIAM Journal on Numerical Analysis 7, 545 (1970).

[36] O. Aberth, Math. Comp. 27, 339 (1973).

[37] R. Descartes, La Géométrie (Ian Maire, Leiden, 1637).

[38] S. Basu, R. Pollack, and M. Coste-Roy, Algorithms in Real Algebraic Geometry, Algorithms and Computation in Mathematics (Springer Berlin Heidelberg, 2013).

[39] M. Mathisson, General Relativity and Gravitation 42, 1011 (2010).

[40] A. Papapetrou, Proc. R. Soc. Lond. A209, 248 (1951).

[41] W. G. Dixon, Royal Society of London Proceedings Series A 314, 499 (1970).

[42] U. Ruangsri, S. J. Vigeland, and S. A. Hughes, Phys. Rev. D94, 044008 (2016), arXiv:1512.00376 [gr-qc].

[43] P. I. Jefremov, O. Yu. Tsupko, and G. S. BisnovatyiKogan, Phys. Rev. D91, 124030 (2015), arXiv:1503.07060 [gr-qc].

[44] S. Hod, Phys. Rev. D88, 087502 (2013), arXiv:1707.05680 [gr-qc]. 